\title{
Anomalous Threshold as the Pivot of Feynman Amplitudes
}

\author{
Stefano Goria ${ }^{\mathrm{a}}$ and Giampiero Passarino ${ }^{\mathrm{a}}$ * \\ ${ }^{a}$ Dipartimento di Fisica Teorica, Università di Torino, Italy
}

Reduction techniques, Landau singularities and differential equations for Feynman amplitudes are briefly reviewed.

\section{Reduction techniques and factorization}

A modern version of reduction of Feynman integrals [1] tells us that

$$
\begin{gathered}
\sum\{\text { 1-loop n-legs Feynman diagrams }\}= \\
\sum_{\mathcal{D}} B_{\mathcal{D}} D_{0}\left(P_{1}^{\mathcal{D}}, \ldots, P_{4}^{\mathcal{D}}\right)+\cdots
\end{gathered}
$$

where $\mathcal{D}$ is a partition of $\{1 \ldots n\}$ into 4 nonempty sets, $P_{i}^{\mathcal{D}}$ is the sum of momenta in $i \in \mathcal{D}$ and $D_{0}$ a scalar box. In other words, scalar one-loop integrals (up to boxes) form a basis. Thus, coefficients in the expansion $\left(B_{\mathcal{D}}\right.$ etc.) are uniquely determined, although some reduction method can be more efficient than others. However, troublesome points where the numerical stability of the result is at stake will always be there. What to do in these cases? We can change (adapt) bases, or avoid bases (expansion).

We explain our idea via examples; first, we consider factorization of Feynman amplitudes, the Kershaw theorem of Ref. [ 2]: any Feynman diagram is particularly simple when evaluated around its anomalous threshold. The singular part of a scattering amplitude around its leading Landau singularity may be written as an algebraic product of the scattering amplitudes for each vertex of the corresponding Landau graph times a certain explicitly determined singularity factor which depends only on the type of singularity (triangle graph, box graph, etc.) and on

\footnotetext{
*Work supported by MIUR under contract 2001023713_006, by INFN and by the European Community's Marie-Curie Research Training Network under contract MRTN-CT-2006-035505 'Tools and Precision Calculations for Physics Discoveries at Colliders'.
}

the masses and spins of the internal particles.

Let us illustrate the consequences of factorization with one example: define a scalar one-loop $N$-leg integral in $n$-dimensions as

$N_{0}^{n}=\lambda_{n} \int \frac{d^{n} q}{\prod_{i=0}^{N-1}[i]}, \quad[i]=P_{i}^{2}+m_{i}^{2}$,

with $\lambda_{n}=\mu^{4-n} /\left(i \pi^{2}\right)$ and $P_{i}=q+\ldots+p_{i}$ $\left(p_{0}=0\right)$. In parametric space we have

$$
\begin{aligned}
N_{0}^{n} & =\left(\frac{\mu^{2}}{\pi}\right)^{N-n / 2} \Gamma\left(N-\frac{n}{2}\right) \mathcal{N}_{0}^{n}, \\
\mathcal{N}_{0}^{n} & =\prod_{i=1}^{N} \int_{0}^{x_{i-1}} d x_{i} V_{N}^{n / 2-N}, \\
V_{N} & =x^{t} H_{N} x+2 K_{N}^{t} x+L_{N}, \quad X_{N}=-K_{N}^{t} H_{N}^{-1} .
\end{aligned}
$$

Standard notation for $N=1,2 \ldots$ is $N_{0}=$ $A_{0}, B_{0} \ldots$; the superscript $n$ will be dropped unless strictly needed. In order to discuss the procedure it is helpful to introduce the following quantities: the BST factor [ [3], $B_{N}=L_{N}-$ $K_{N}^{t} H_{N}^{-1} K_{N}$, the Gram matrix, $H_{N, i j}=-p_{i} \cdot p_{j}$ the Caley matrix [4]

$M_{N}=\left(\begin{array}{ll}H_{N} & K_{N} \\ K_{N}^{t} & L_{N}\end{array}\right)$

It follows that [ [5] $B=C / G$ for any $N$, where $C=\operatorname{det} M$ and $G=\operatorname{detH}$. Landau singularities are seen as pinches (we assume that masses and invariants $\in R$ ) when we write $V_{N}=$ $\left(x-X_{N}\right)^{t} H\left(x-X_{N}\right)+B_{N}$. This realtion indeed shows that $B_{N}=0$ is the origin of the pinch on the integration contour at the point of coordinates $x=X_{N}$; therefore, if the conditions, 
$B_{N}=0$ and $0<X_{N, N-1}<\ldots<X_{N, 1}<1$, are satisfied we will have the leading singularity of the diagram (hereafter AT).

Nowadays, the keyword in any reduction procedure is to avoid inverse powers of Gram determinants. A common wisdom, but why? The vanishing of the Gram determinant is the condition for the occurrence of non-Landau singularities, connected with the distorsion of the integration contour to infinity; furthermore, for complicated diagrams (see Sect. 10 of Ref. [ 6]), there may be pinching of Landau $(C=0)$ and non-Landau singularities $(G=0)$, giving rise to a non-Landau singularity whose position depends upon the internal masses [ 7 .

Given the above properties, the factorization of Kershaw theorem [2] follows. The beauty of being at the anomalous threshold is that scalar products are frozen as a consequence of the Landau equations and the amplitude factorizes. Therefore, the AT looks perfect for boundary conditions, as long as it is inside the physical region. Alternatively we may expand and match residues at a given AT [1].

Let us consider standard reduction [8] as compared to modern techniques [9] by taking a box diagram with $q \cdot p_{1}$ in the numerator:

$D \cdot p_{1}=\sum_{i=1}^{3} D_{1 i} p_{1} \cdot p_{i}=-\sum_{i=1}^{3} D_{1 i} H_{1 i}$.

A careful application of the standard method gives

$D_{1 i}=-\frac{1}{2} H_{i j}^{-1} d_{j}, \quad d_{i}=D_{0}^{(i+1)}-D_{0}^{(i)}-2 K_{i} D_{0}$,

where $D_{0}^{(i)}$ is the scalar triangle obtained by removing propagator $i$ from the box. Therefore we obtain

$D \cdot p_{1}=\frac{1}{2} \sum_{i, j=1}^{3} H_{i j}^{-1} H_{1 i} d_{j}=\frac{1}{2} d_{1}$,

without having to introduce $G_{3}$. Furthermore, the coefficient of the scalar $D_{0}$ in the reduction is $1 / 2\left(m_{0}^{2}-m_{1}^{2}-p_{1}^{2}\right)$. At the AT of the box we must have $q^{2}+m_{0}^{2}=0,\left(q+p_{1}\right)^{2}+m_{1}^{2}=0$, etc. Therefore the coefficient of $D_{0}$ is fixed by

$\left.2 q \cdot p_{1}\right|_{\text {AT }}=m_{0}^{2}-m_{1}^{2}-p_{1}^{2}$, which is what a careful application of standard reduction gives. Note that one gets the coefficient without having to require a physical singularity. In standard reduction for a $N$-point function each, reducible, scalar product in the numerator is replaced by a difference of propagators plus a $K$-factor. The latter is what is predicted by factorization at the anomalous threshold; the procedure is continued and one finds $N-1$ point functions with reducible and also irreducible scalar products; for the latter inverse powers of Gram determinants remain.

It is worth noting that starting from six legs factorization must be understood as performed at some subLeading Landau singularity of the diagram [10, which is easily achieved by using the BST-algorithm [ 3]. If the derivation is to hold we must further require that the leading Landau singularity point does not also lie on the Landau curve of its sub-graphs. For illustration, consider a box in $n$-dimensions in a region where $B_{4} \neq 0$. BST relations allow us to decompose the box in a $n+2$-dimensional box plus four $n$-dimensional triangle, $D^{n} \rightarrow D^{n+2} \oplus C^{n}$. A second application gives $D^{n+4} \oplus C^{n+2} \oplus C^{n}$. A box in 8 -dimensions as well as a triangle in 6 -dimensions cannot develop a singularity, threfore the subleading singularities of the original box are given by the leading ones of the four triangles obtained by shrinking one of the lines in the box to a point. The coefficients of the decomposition can be found in [5] and the argument can be generalized to arbitrary number of legs.

To summarize, at least in one point we can avoid reduction, all integrals are scalar; however, we need to have the AT inside the physical region $R_{\text {phys }}$ (support of $\Delta^{ \pm}$-propagators in $R$ ) Since this is a rare event we must have a generalization of the factorization theorem: prove that the AT, even with invariants $\notin R_{\text {phys }}$ implies a frozen $q$.

If a one-loop, $N$-legs scalar diagram is singular at $x=X_{N} \in R$ then consider $N^{n \mu} p_{\mu l}$,

$$
\begin{gathered}
N^{n} \cdot p_{l}=-\sum_{i=1}^{N} \mathcal{N}^{n}(i) p_{l} \cdot p_{i} \\
\sum_{i=1}^{N} \mathcal{N}^{n}(i) H_{l i} \underset{A T}{\sim} \sum_{i=1}^{N} \mathcal{N}^{n}(1) H_{l i} X_{i}=-K_{l} \mathcal{N}_{n} .
\end{gathered}
$$


where $\mathcal{N}^{n}(i)$ is the same as the scalar integral $\left(\mathcal{N}^{n}(1)\right)$ but with one power $x_{i}$ in the numerator, and $H X=-K$ : this leads to generalized factorization since, at the AT, all scalar products are replaced by the solution of $\left(q+\ldots+p_{i}\right)^{2}+m_{i}^{2}=0$, with $i=0, \ldots, N-1$.

\section{Feynman diagrams aroud AT}

In this section we consider a classification of physical ATs: for instance, direct calculation shows that, for $N=4$, there are 14 branches in $p$-(real) space. In general, this classification is much easier when we use the Coleman - Norton theorem [11]. As a consequence of it, in a $2 \rightarrow 2$ process, two unstable particles in the initial state are needed. Other simple examples of physical AT are represented by a) $\gamma^{*}\left(Z^{*}\right) \rightarrow \bar{b} b H$ (for a virtuality $s>4 m_{t}^{2}$ and $M^{2}(H)>4 M_{W}^{2}$ ) and b) from pentagons arising in the reduction of the hexagon in $\gamma^{*}\left(Z^{*}\right) \rightarrow \bar{b} b \bar{\nu} \nu H$ (as suggested by A. Denner).

The expansion of Feynman integrals around their AT is easy to derive analytically and only requires Mellin-Barnes and sector decomposition techniques as explained in Ref. [5]. Examples of leading behavior are: for the vertex $C_{0} \sim \ln B_{3}$; for the box $D_{0} \sim B_{4}^{-1 / 2}$; for the pentagon $E_{0} \sim$ $B_{5}^{-1}$ and no singularity for the hexagon $F_{0}$ in 4 dimensions [ 10]; e.g. $\operatorname{Im} C_{0}$ has a logarithmic singularity, $\operatorname{Re} C_{0}$ has a discontinuity. Here we do not consider infrared/collinear configurations where we expect an enhancement of the singular behavior (in the residues of IR/coll. poles).

It is worth noting the non-integrable (scalar) pentagon singularity which seems to require the introduction of complex masses for unstable internal particles [12]. For integrable singularities we always average over a Breit-Wigner of the invariant mass of unstable external particles.

\section{Differential equations}

An interesting feature of factorization at AT is the possibility of introducing a differential equation with boundary conditions at the AT where the amplitude is directly given in terms of scalar functions; what we want is an ODE for the full amplitude, with real momenta and one boundary condition; this requires to find the right variable. The advantages of this procedure are given by a total absence of reduction and by the extedibility to higher loops.

It is well-known that non-homogeneous systems of ODE [13] are easy to obtain with IBPtechniques [14] but the non-homogeneous part requires (a lot) of additional work; the natural alternative would be to introduce PDE. They are notoriously much more difficult to handle even if homogeneous (compatible) systems of nth-order PDE are easy to derive, a fact that has to do with the hypergeometric character of one-loop diagrams. It is enough to use Kershaw expansion around pseudo-threshold [15] and a generalization of Horn-Birkeland-Ore theory [16].

\section{Diffeomorphisms}

Let us restrict to ODE. To achieve our goal we find it most natural to introduce special diffeomorphisms $\mathcal{T}$ of the Feynman diagrams. Define $P_{i}(z)=T_{i j}(z) p_{j}$ with $\sum P_{i}=\sum p_{i}=0$ and with $T_{i j}(0)=\delta_{i j}$; next we look for a $z=$ $z_{A T} \in R$ where the transformed diagram is singular. Furthermore, $\mathcal{T}$ is physical if maps $D(0)$ onto a $D(z)$ which is singular at $z_{A T} \in R$ and $s_{i j} \rightarrow S_{i j}(z) \in \mathrm{Phys}_{z}$, where $s_{i j}$ and $S_{i j}$ are invariants; no restriction on $s_{i j}$ is required. $\mathcal{T}$ is unphysical if maps $D(0)$ onto a $D(z)$ which is singular at $z_{A T} \in R$ but $s_{i j} \rightarrow S_{i j}(z) \notin$ Phys $_{z}$; it requires restrictions on the original invariants $s_{i j}$.

A general solution of our problem is as follows: if $\exists$ a diagram $\bar{D}$, a transformation $\bar{T}$ such that $\bar{D}(z)=\bar{T}(z) \bar{D}$ with $\bar{T}(0)=I$ and $\bar{D}\left(z_{A T}\right)$ singu$\operatorname{lar}\left(z_{A T} \in R\right)$ then we map $D$ as follows:

$$
\begin{aligned}
D & \rightarrow D\left(z, z_{A T}\right) \\
D\left(z, z_{A T}\right) & =T_{1}\left(z, z_{A T}\right) D+T_{2}\left(z, z_{A T}\right) \bar{D}(0) \\
T_{1}\left(0, z_{A T}\right) & =I, \quad T_{2}\left(0, z_{A T}\right)=0 \\
T_{1}\left(z_{A T}, z_{A T}\right) & =0, \quad T_{2}\left(z_{A T}, z_{A T}\right)=I .
\end{aligned}
$$

It is worth mentioning that, in this way, we can write a differential equation for the full amplitude instead of one for each master integral with different boundary conditions. The interesting feature can be summarized as follows: for a given topol- 
ogy which is candidate to satisfy Coleman - Norton (e.g. crossed box in $2 \rightarrow 2$ ) we perform the transformation in such a way that the new invariants indeed satisfy the conditions of the theorem; for all parent topologies (e.g. direct boxes) we use the general mapping described in Eq.(6).

It is straightforward to see how our approach is related to the one of differential equations in Mandelstam variables:

$\tau_{i j}^{-1} \frac{d}{d z}=O_{i j}(\{s\})=P_{i \mu} \frac{\partial s_{l}}{\partial P_{j \mu}} \frac{\partial}{\partial s_{l}}$,

where $\tau=(d T / d z) T^{-1}$.

As an example for a four-point function we consider one of the crossed diagrams in $g g \rightarrow \gamma \gamma$ with a massive loop. The transformation is

$T=\left(\begin{array}{cccc}1-z & 0 & z & 0 \\ 0 & 1-z & 0 & z \\ z & 0 & 1-z & 0 \\ 0 & z & 0 & 1-z\end{array}\right)$

The transformed invariants are $M_{i}^{2}=z(1-z) u$ and

$S=(1-2 z)^{2} s, \quad T=(1-2 z)^{2} t, \quad U=u$.

The solution of $B_{4}=0$ which makes singular the integrand is

$$
\begin{aligned}
& 2 u^{2} z_{A T}\left(z_{A T}-1\right)=4 m^{2} s+u t \\
+ & {\left[s\left(4 m^{2}-u\right)\left(4 m^{2} s+u t\right)\right]^{1 / 2} . }
\end{aligned}
$$

The effect of the transformation is simple, we have mapped the original box onto a box which satisfies the condition stated in Coleman - Norton theorem.

As an example of ODE in $z$ we consider the scalar box after the transformation $P_{1,4}=p_{1,4}+$ $z\left(p_{1}+p_{2}\right)$ and $P_{2,3}=p_{2,3}-z\left(p_{1}+p_{2}\right)$,

$$
\begin{aligned}
D_{0}^{n}(\{\nu\}) & =\lambda_{n} \int d^{n} q \frac{1}{\prod_{i=0,3}[i]^{\nu_{i}}}, \\
D_{0}^{n}(i) & =D_{0}^{n}(1, \ldots, 2, \ldots, 1) \\
D_{0}^{n} & =D_{0}^{n}(1, \ldots, 1) \\
\frac{d}{d z} D_{0}^{n} & =2 z s\left[D_{0}^{n}(2)+D_{0}^{n}(4)\right]+\text { triangles }
\end{aligned}
$$

Using IBP-techniques (and dropping the superscript $n$ ) we derive

$D_{0}(i)=R_{4, i j}^{-1} d_{j}, \quad \operatorname{det} R_{4}\left(z_{A T}\right)=0$ where $d_{i}$ contains $D_{0}$ or triangles. Introducing $r=z^{2}-z$, we obtain

$\frac{d}{d r} D_{0}(r)=C_{4}^{-1}(r)\left[X(r) D_{0}(r)+D_{\text {rest }}(r)\right]$

where $C_{4}$ is the corresponding Caley determinant. Furthermore, we have

$\frac{d}{d r} C_{4}=-2 X(r)$,

which leads to the expected solution,

$D_{0}(r)=\frac{D^{\text {sing }}}{\left(r-r_{A T}\right)^{1 / 2}}+D^{\text {reg }}(r)$

Before turning to a final example it is instructive to consider the deep connection between ODE for Feynman diagrams, IBP identities and analytical properties of the diagrams. It can be seen as follows: for a given set of momenta we consider the transformation $P_{i}=T_{i j}(z) p_{j}$, subject to $\sum P=\sum p=0$. Consider a generalized, scalar, box (arbitrary powers in propagators); we will also need the IBP equations for $D_{0}(1,1,1,1)$ and will define $D_{0}(1)=D_{0}(1,2,1,1)$ till $D_{0}(4)=D_{0}(2,1,1,1)$. Again, we can use IBP to get

$R_{4 ; i j} D_{0}(j)=\delta_{i 4} D_{0}(1,1,1,1)+\Delta D_{0}(i)$,

where $\Delta D_{0}(i)$ contains only 3 -point functions. Introduce the Caley determinant $C_{4}$; it follows that $R_{4} U=2 M_{4}$, where $U$ is unimodular (a similar relation holds for arbitrary $N$ ), i.e.

$\operatorname{det} R_{4}=16 C_{4}$,

so that the differential equation for the transformed box is

$\frac{d}{d z} D_{0}(1,1,1,1)=\frac{X}{C_{4}} D_{0}(1,1,1,1)+Y$.

A straightforward calculation shows that

$X=-\frac{1}{2} \frac{d}{d z} C_{4}$

for all values of $\{p\},\{m\}$ and for an arbitrary transformation $T$. Eq. (14) holds for all $N$, i.e. $\operatorname{det} R_{N}=2^{N} C_{N}$, where one should remember 
that in four dimensions $C_{N}=0$ for $N>6$. The homogeneous term has the general stucture

$$
\begin{aligned}
\frac{d}{d z} N_{0}^{n} & =-\frac{1}{2}\left[C_{N}^{-1} \frac{d C_{N}}{d z}\right. \\
& \left.+(N-n) B_{N}^{-1} \frac{d B_{N}}{d z}\right] N_{0}^{n}+Y_{N-1}
\end{aligned}
$$

where $Y_{N-1}$ is a combination of $N-1$-point integrals.

\section{An explicit example}

Our last case in point is given by the ODE for $H \rightarrow g\left(p_{1}\right) g\left(p_{2}\right)$ decay amplitude. Here there is one form factor $F_{D}$ that can be written, without reduction, as $F_{D}=\sum_{i} F_{i}$,

$$
\begin{aligned}
F_{1} & =\frac{\lambda_{n}}{2} \int d^{n} q \frac{M_{H}^{2}-2 m_{t}^{2}}{[0][1][2]} \\
F_{2} & =-2 \lambda_{n} \int d^{n} q \frac{q \cdot p_{1}}{[0][1][2]} \\
(n-2) F_{3} & =\lambda_{n} \int \frac{d^{n} q}{[0][1][2]}\left[(6-n) q^{2}\right. \\
& \left.+\frac{16}{M_{H}^{2}} q \cdot p_{1} q \cdot p_{2}\right]
\end{aligned}
$$

Suppose that $M_{H}^{2}<4 m_{t}^{2}$ : define the transformation $P_{i}=T_{i j} p_{j}$ with

$T=\left(\begin{array}{cc}z & 1-z \\ 1-z & z\end{array}\right)$

Then $B \rightarrow M_{H}^{2} C / G$ with $C=r^{2}+\mu_{t}^{2}(1+4 r)$ and $G=-\frac{1}{4} M_{H}^{2}(1+4 r)$, being $r=z(z-1)$ and $\mu_{t}^{2} M_{H}^{2}=m_{t}^{2}$. The solution for AT is given by

$$
\begin{aligned}
& r_{A T}=-2 \mu_{t}^{2}\left[1+\sqrt{1-\frac{1}{4 \mu_{t}^{2}}}\right] \\
& -\infty<r_{A T}<-\frac{1}{2}
\end{aligned}
$$

The corresponding system of ODE will be written in terms of $F_{1,2}$ and $F_{D}$ giving

$\frac{d}{d r} F_{i}=X_{i j} F_{j}+Y_{j}, \quad i, j=1,2, D$,

where $X$ and $Y$ are obtained by using IBP techniques, e.g.

$X_{D 1}=X_{D D}=-\frac{2}{1+4 r}, \quad X_{D 2}=0$, etc, with $Y$ given by generalized two-point functions. Boundary conditions at AT are

$$
\begin{aligned}
& F_{D} \underset{A T}{\tilde{A}} {\left[\frac{M_{H}^{2}}{8}\left(1+6 r_{A T}\right)\right.} \\
&\left.-m_{t}^{2}\left(1+4 r_{A T}\right)\right] C_{0}^{\operatorname{sing}}(z), \\
& F_{1} \underset{A T}{ } \frac{1}{2}\left(M_{H}^{2}-2 m_{t}^{2}\right) C_{0}^{\operatorname{sing}}(z) \\
& F_{2} \tilde{A T} M_{H}^{2} z_{A T} C_{0}^{\operatorname{sing}}(z) .
\end{aligned}
$$

More general transformations, not singular for any $z \in R$, exist but will not discussed here. The pure-scalar term becomes

$$
\begin{aligned}
C_{0}(r) & =C_{0}^{\text {sing }}(r)+c_{0}^{\mathrm{reg}}(r) \\
& =c_{0}^{\mathrm{sing}}(r) \ln \frac{B_{3}(r)}{M_{H}^{2}}+c_{0}^{\mathrm{reg}}(r) \\
\frac{d}{d r} c_{0}^{\text {sing }} & =-\frac{2}{1+4 r} c_{0}^{\text {sing }}
\end{aligned}
$$

with boundary conditions

$c_{0}^{\operatorname{sing}}\left(z_{A T}\right)=\frac{2 \pi i}{M_{H}^{2}} \beta\left(r_{A T}\right) \quad \beta^{2}(r)=1-4 \frac{\mu_{t}^{2}}{r}$

while the regular part is computed numerically (boundary condition for the regular part will not be reported here).

The general strategy, e.g. for processes with $N=4$, is as follows: define

$D_{n_{0} \ldots n_{3}}(i)=\lambda_{n} \int d^{n} q \frac{(q \cdot q)^{n_{0}} \ldots\left(q \cdot P_{3}\right)^{n_{3}}}{[0] \ldots[i]^{2} \ldots[3]}(22)$

which satisfy a recurrence relation (IBP)

$D_{n_{0} \ldots n_{3}}(i)=R_{i j}^{-1} d_{n_{0} \ldots n_{3}}(j)+d_{n_{0} \ldots n_{3}}^{\prime}(i)$,

then find the minimal set of linear combinations $F=c D$ such that Amp $=\sum F$ with $\{F\}$ closed under $d / d z$.

\section{Extension to multi-loop}

Although we shall not discuss higher loops in details here, we present one simple example: the equal mass two-loop sunset $S$ [17]; with scaled masses $m=1$ and $p^{2}=x$ we perform the transformation $x \rightarrow z x$

$$
\begin{aligned}
& x z(x z+1)(x z+9) \frac{d^{2}}{d z^{2}} S(x, z)= \\
& P(x, z) \frac{d}{d z} S(x, z)+Q(x, z) S(x, z)+R(x, z)
\end{aligned}
$$


The AT solution is $z_{A T}=-x^{-1} \quad$ (note that here $\mathrm{AT}=$ pseudo-threshold). For different masses we map

$m_{i} \rightarrow M_{i}=\frac{z-z_{A T}}{1-z_{A T}} m_{i}+\frac{1-z}{1-z_{A T}} m$,

and use the previous calculation of AT.

\section{Conclusions}

In conclusions we have presented a proposal for solving the problem of reducing Feynman diagrams which is based on a single variable deformation of the amplitude.

\section{REFERENCES}

1. F. Cachazo, arXiv:0803.1988 [hep-th].

2. D. Kershaw, Phys. Rev. D 5, 1976 (1972).

3. J. Bernstein, Modules over a ring of differential operators, Functional Analysis and its Applications 5(1971); M. Sato, Nagoya Mat. J. 120 (1990) 1;

S. C. Coutinho, A Primer of Algebraic DModules, LMS Student Text 33 (1995); ; F. V. Tkachov, Nucl. Instrum. Meth. A 389 (1997) 309 hep-ph/9609429; G. Passarino, Nucl. Phys. B 619 (2001) 257 arXiv:hep-ph/0108252.

4. D. B. Melrose, Nuovo Cim. 40 (1965) 181.

5. A. Ferroglia, M. Passera, G. Passarino and S. Uccirati, Nucl. Phys. B 650, 162 (2003) arXiv:hep-ph/0209219.

6. Z. Bern et al. [NLO Multileg Working Group], arXiv:0803.0494 [hep-ph].

7. R.J. Eden, P.V. Landshoff, D.I. Olive, and J.C. Polkinghorne, The Analytic S-Matrix, Cambridge Univ. Press, 1966; C. Hua and V. L. Teplitz, Homology and Feynman integrals, W. A. Benjamin, New. York 1966.

8. G. Passarino and M. J. G. Veltman, Nucl. Phys. B 160 (1979) 151.

9. Z. Bern, G. Chalmers, L. J. Dixon, D. C. Dunbar and D. A. Kosower, arXiv:hep-ph/9503261.

10. P. V. Landshoff, Nucl. Phys. 20 (1960) 129.

11. S. Coleman and R. E. Norton, Nuovo Cim. 38, 438 (1965).
12. A. Denner and S. Dittmaier, Nucl. Phys. Proc. Suppl. 160, 22 (2006) arXiv:hep-ph/0605312; S. Actis and G. Passarino, Nucl. Phys. B 777, 100 (2007) arXiv:hep-ph/0612124.

13. M. Argeri and P. Mastrolia, Int. J. Mod. Phys. A 22, 4375 (2007) arXiv:0707.4037 [hep-ph]].

14. F.V. Tkachov, New methods for evaluation of multi-loop Feynman diagrams, PhD thesis, INR, Moscow, Russia, 1984;

F.V. Tkachov, Phys. Lett. B 100 (1981) 65; K.G. Chetyrkin and F.V. Tkachov, Nucl. Phys. B 192 (1981) 159.

15. D. S. Kershaw, Phys. Rev. D 8, 2708 (1973).

16. A. Erdelyi et al., Higher Transcendental Functions, vol. 2, Bateman Manuscript Project, McGraw-Hill, 1953.

17. E. Remiddi, Nucl. Phys. Proc. Suppl. 135, 247 (2004). 